\title{
Synthesis of Well-Defined Three-Arm Star-Branched Polystyrene through Arm-First Coupling Approach by Atom Transfer Radical Polymerization
}

\author{
Syed Shahabuddin, Fatem Hamime Ismail, \\ Sharifah Mohamad, and Norazilawati Muhamad Sarih \\ Polymer Research Laboratory, Chemistry Department, Faculty of Science, University of Malaya, 50603 Kuala Lumpur, Malaysia \\ Correspondence should be addressed to Norazilawati Muhamad Sarih; nmsarih@um.edu.my
}

Received 23 August 2014; Revised 23 December 2014; Accepted 25 December 2014

Academic Editor: Saad Khan

Copyright ( 2015 Syed Shahabuddin et al. This is an open access article distributed under the Creative Commons Attribution License, which permits unrestricted use, distribution, and reproduction in any medium, provided the original work is properly cited.

Here we describe a simple route to synthesize three-arm star-branched polystyrene. Atom transfer radical polymerization technique has been utilized to yield branched polystyrene involving Williamson coupling strategy. Initially a linear polymeric chain of predetermined molecular weight has been synthesized which is further end-functionalized into a primary alkyl bromide moiety, a prime requisition for Williamson reaction. The end-functionalized polymer is then coupled using 1,1,1-tris(4hydroxyphenyl)ethane, a trifunctional core molecule, to give well-defined triple-arm star-branched polystyrene.

\section{Introduction}

Facile and simple experimental conditions of radical polymerization make atom transfer radical polymerization (ATRP) a technique of significant current interest. In the short duration of a decade, ATRP technique has been successfully explored and its mechanism has been completely elucidated by various researchers [1-3]. Due to its simplicity, versatility, and cost effectiveness, ATRP has become one of the most powerful techniques for the synthesis of narrow molecular weight polymers [4] and copolymers $[5,6]$, even though several researchers have reported other controlled radical polymerization methods $[7,8]$. The synthesis of polymers with controlled molecular architecture including chain branching is also an area of ongoing interest in both academic and industrial research laboratories since branching is a key feature which may control the physical properties of the polymeric material. A common goal for the polymer chemist involved in the synthesis of polymers with controlled branched architectures is to control molecular parameters such as molecular weight, distribution of molecular weight, dispersity (Đ), and branching pattern, as these parameters have a significant influence on the physical properties of the polymer. The development of living polymerization mechanisms paved the way to achieve these goals; however, the sensitivity of living polymerization techniques such as anionic, cationic, and group transfer polymerization towards oxygen, moisture, and impurities makes its use challenging and limits its use to the polymerization of nonfunctional monomers. Atom transfer radical polymerization (ATRP) is a technique which has seen significant interest since it was first established in the mid-1990s and has seen significant advances including various metal catalyzed radical polymerization techniques since this time $[9,10]$. With a pronounced tolerance to water and protonic species ATRP can be carried out easily and can be applied to a very wide variety of functional monomers. For macromolecular engineering, ATRP is an effective technique which can be utilized to tailor desired properties.

Star polymers with multiple linear chains attached to a core epitomize the simplest branching topology [11]. Star polymers exhibit physical properties which are distinctly different in many respects to their linear analogues making them ideal material for various potential applications. Various polymerization techniques have been utilized for preparing a large variety of star polymers with distinct 
chemical compositions. These star-branched polymers are synthesised by one of two major synthetic routes, namely, core-first and arm-first methods. A multifunctional core initiates chain growth of the arms of the star in the core-first approach, whereas before further polymerization branching is introduced at the termini of arms. For the synthesis of regular stars shaped polymers, this technique has been proven very effective and sequences of well-defined star polymers with precise arm numbers have been fruitfully synthesised by atom transfer radical polymerization (ATRP) [1214], ring-opening polymerization (ROP) [15-17], reversible addition fragmentation chain transfer (RAFT) [18], anionic polymerization $[19,20]$, and group transfer polymerization [21]. In the arm-first approach, the arms of the star are prepared first and the polymers are then coupled efficiently to multifunctional core molecules. The polymeric arms can be end-functionalized with halides, azides, or alkynes and can be coupled efficiently via Williamson coupling, azide coupling, or click coupling reactions [22-24].

Recently, notable achievements have been made by Hutching et al. [25, 26], Hirao et al. [20, 27], Gnanou et al. [28, 29], Hadjichristidis et al. [30, 31], Trollsås and Hedrick [32], and Monteiro et al. [23] in the synthesis of well-defined dendritically branched polymers. Hutchings et al. recently reported the synthesis of dendritically branched polystyrene, which they call Dendrimacs and HyperMacs, through convergent approach using Williamson coupling reactions. Hirao et al. reported the synthesis of poly(methyl methacrylate) (PMMA) dendritic polymers by coupling endfunctionalized living anionic PMMA chains with benzyl bromide moieties through divergent approach. Using end group modified dendritic PMMA, up to seven generations of branching was obtained. A divergent approach has been utilized by Gnanou et al. for the synthesis of dendrimer-like polystyrene through ATRP. They were successful in achieving third-generation branched dendritic polystyrene using multifunctional initiators based on calixarene. Hadjichristidis et al., based upon stepwise convergent methodology via anionic polymerization and coupling based on chlorosilane reactions, has synthesised well-defined second- and thirdgeneration dendrimer-like polymers. A parallel divergent strategy has been reported by Trollsås and Hedrick where they described the sysnthesis of well-defined polymeric structures with the living ring-opening polymerization (ROP) of ecaprolactone. A unique combination of atom transfer radical polymerization (ATRP) and coupling reactions involving click chemistry in a convergent coupling methodology has been described by Monteiro et al. for the synthesis of dendritic polymers.

In the present contribution, the synthesis of a threearm star-branched polymer of polystyrene has been reported by involving only two simple reaction steps, as shown in Schemes 1 and 2 . Synthesis of the linear polystyrene with controlled molecular weight in homogenous reaction medium using DMF (10\% v/v) [33] through ATRP and transformation of controlled/living radical polymeric chains into an alkyl brominated chains is the first step. Conversion of alkyl brominated linear polystyrene involving effective Williamson coupling reaction with a trifunctional core to give a triarm branched star shape polymer is the second and the final step. The simplicity of the reaction steps makes it an effective tool for the production of branched polymers.

\section{Experimental Section}

2.1. Materials. Styrene (Sigma Aldrich, >99\%) was dried with calcium hydride and degassed in vacuum lines by several freeze-pump-thaw cycles and distilled freshly into the reaction vessel immediately before use. Cesium carbonate (Acros Organics, 99.99\%) was dried in a vacuum oven and stored in a desiccator. 1-Bromoethyl benzene (initiator) (97\%), copper(I) bromide (catalyst) (99.99\%), 2,2dipyridyl (ligand) (99\%), 1,1,1-tris(4-hydroxylphenyl)ethane (99\%), and 3-bromo-1-propanol (97\%) were used as received from Sigma Aldrich. Solvents utilized for the purification and characterization of polymer, namely, toluene (Sigma Aldrich) and methanol (Fisher), were used as received.

2.2. Measurements. Molecular weight determination was done by gel permeation chromatography (GPC, Waters 590) with a $105,104,103,500$, and $100 \AA$ set of microstyragel columns equipped with refractive index, multiangle light scattering, and viscosity detectors with polystyrene standard. A value of $0.1870 \mathrm{~mL} / \mathrm{g}$ was used as the $\mathrm{dn} / \mathrm{dc}$ of polystyrene both for the calibration and the analysis of prepared polymers. Filtered THF (HPLC grade) was used as an eluent with a flow rate of $1.0 \mathrm{~mL} / \mathrm{min}$ at $25^{\circ} \mathrm{C}$. Samples $(1 \mathrm{mg} / \mathrm{mL})$ were prepared in THF and filtered prior to injection using $0.45 \mu \mathrm{m}$ teflon filters.

${ }^{1} \mathrm{H}$ NMR spectra were obtained on Bruker AVN $400 \mathrm{MHz}$ spectrometer for polymer samples using deuterated chloroform (Merck).

2.3. Synthesis of Alkyl Brominated Polystyrene. The linear polymeric chains were made by ATRP in presence of DMF. The catalyst, copper(I) bromide $\left(0.15 \mathrm{~g}\right.$ or $\left.1.044 \times 10^{-3} \mathrm{~mol}\right)$, ligand 2,2-dipyridyl $\left(0.41 \mathrm{~g}\right.$ or $\left.2.61 \times 10^{-3} \mathrm{~mol}\right)$, dry DMF $(1.2 \mathrm{~mL}$ or $10 \% \mathrm{v} / \mathrm{v}$ with respect to monomer), and a magnetic stirrer were introduced in a attachable flask fitted with rubber septum. To make the flask oxygen- and moisture-free, it was purged with nitrogen followed by vacuum cycle several times. Deoxygenated styrene $\left(10.88 \mathrm{~g}\right.$ or $\left.10.44 \times 10^{-2} \mathrm{~mol}\right)$ was then added to flask using gas tight glass syringe and the resulting solution was stirred at room temperature until it becomes homogeneous. The flask was immersed in an oil bath at $110^{\circ} \mathrm{C}$ and 1-bromoethyl benzene $(0.26 \mathrm{~g}$ or 1.044 $\times 10^{-3} \mathrm{~mol}$ ) as initiator was added to the reaction mixture using glass syringe. A sample of polymer solution was taken after 3 hours and was terminated with degassed methanol for molecular weight and NMR analysis. The polymer obtained from this sample was subjected to coupling using Williamson coupling agent, 1,1,1-tris(4-hydroxyphenyl)ethane (10 mol \% with respect to polymer) using cesium carbonate as base and DMF as solvent in inert nitrogen atmosphere. Small samples were withdrawn at specific time interval to monitor the progress of coupling reaction. To the rest of the controlled/living polymer solution, 1-bromo-3-propanol 


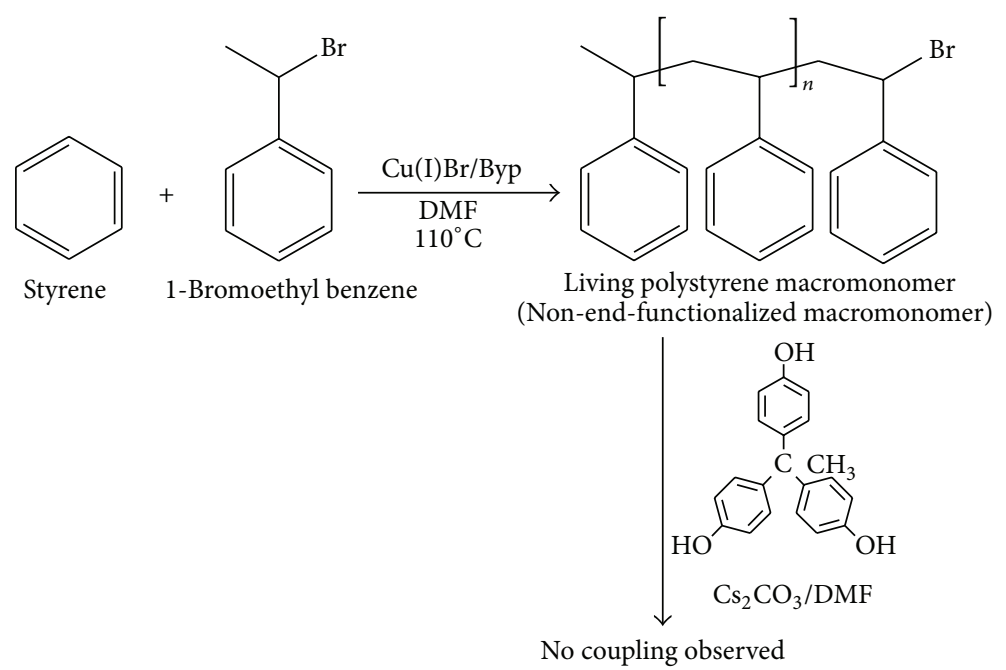

SCHEme 1: Synthesis of branched polystyrene without end functionalization.

$\left(0.15 \mathrm{~g}\right.$ or $\left.1.044 \times 10^{-3} \mathrm{~mol}\right)(1: 1$ with respect to initiator $)$ was added and the solution was stirred at $60^{\circ} \mathrm{C}$ for 24 hours. The reaction was terminated using degassed methanol. The reaction mixture was then diluted using THF and catalyst was removed by filtration through neutral alumina. The polymer was recovered by precipitation in methanol, redissolved in THF and reprecipitated in methanol, and dried in vacuum oven at $70^{\circ} \mathrm{C}$ overnight. Yield $>80 \%, \mathrm{Mn}=7455 \mathrm{~g} \cdot \mathrm{mol}^{-1} \mathrm{PDI}$ $=1.17$.

2.4. Coupling of End-Functionalized Polystyrene through Williamson Reactions. An inert nitrogen atmosphere was used to carry out Williamson coupling reaction. Alkyl brominated polystyrene (Mn $\left.7455 \mathrm{~g} \cdot \mathrm{mol}^{-1}, 2.68 \times 10^{-4} \mathrm{~mol}\right), 1,1,1-$ tris(4-hydroxyphenyl)ethane $\left(8.5 \mathrm{mg}, 2.80 \times 10^{-5} \mathrm{~mol}\right)$, and cesium carbonate $\left(0.46 \mathrm{~g}, 1.4 \times 10^{-3} \mathrm{~mol}\right)$ were dissolved in $10 \mathrm{~mL}$ of DMF. The solution was immersed in an oil bath maintained at $60^{\circ} \mathrm{C}$ and stirred vigorously. Small samples were taken out at specific time interval to monitor the progress of coupling by GPC until no additional rise in molecular weight was witnessed. The solution was then cooled to room temperature, precipitated in methanol, and further purified, using toluene and methanol as solvent and nonsolvent system, by fractionation.

\section{Results and Discussion}

Initially, as per Scheme 1, the star-shaped polymer synthesis was attempted directly using the linear polymer obtained from ATRP as these polymers are brominated polymers. Based upon the structure of initiators in ATRP, the end groups of the polymers are defined. The alkyl group of the alkyl halide initiator is attached at one end of the polymeric chain whereas the halogen atom is shifted to the other end of the polymeric chain [34]. However, the result from the coupling of such polymer with a trifunctional core was not as expected. The coupling was inefficient and there was no apparent increase in the molecular weight of the resulting polymers as seen from the GPC result (Figures 4(a) and 4(b)). Both peaks appeared nearly at the same retention time with only a little difference as can be seen from Figure 4(b) where there was a trace of high molecular weight shoulder (for coupled polymer) indicating the inefficient coupling reaction. The cause of this inefficient coupling was then analyzed and it was found that, for efficient Williamson coupling between an alkyl halide and alcoholic functionality, the alkyl halide must be primary with at least two to three primary carbon chains [35]. Therefore, the end group has to be modified to introduce a primary alkyl bromide moiety at the end of the polymeric chain as per Scheme 2 . Thus at the end of polymerization reaction, as monitored by GPC, a slight excess of 1-bromo-3-propanol (1:1 ratio with respect to initiator) was introduced into the mixture of the living polymer solution. The end functionalization was investigated using ${ }^{1} \mathrm{H}$ NMR. As shown in Figure 2, new signals at 3.75 (from $\mathrm{CH}_{2} \mathrm{Br}$ ) were recorded in ${ }^{1} \mathrm{H}$ NMR spectrum, which were absent in nonalkyl brominated polystyrene (Figure 1). Thus a primary alkyl bromide chain was successfully incorporated into the linear polymeric chain for efficient Williamson reaction.

The coupling reaction between the alkylbromide moiety on the linear polystyrene chain with Williamson coupling agent, a multifunctional core, 1,1,1-tris(4-hydroxyphenyl)ethane (10 mol \% with respect to polymer), was carried out in the presence of cesium carbonate as base, to yield starbranched polymer. This reaction was carried out in inert nitrogen atmosphere using dry DMF as solvent since DMF has proven to be a good solvent for Williamson coupling using bromine as leaving group and cesium carbonate as base [25]. The GPC results show significant increase in molecular weight as the peak shifted towards lower retention time (Figure 4(c)) and molecular weight becomes approximately tripled. The crude coupled polymer also contained some uncoupled polymer which was evident from the low molecular weight shoulder in GPC traces (Figure 4(c)). The coupled branched polymer obtained was then subjected to 


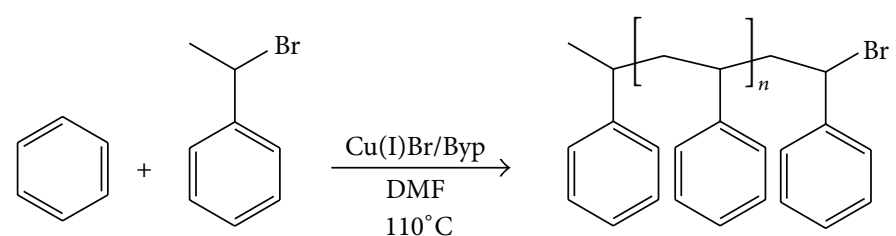

Styrene 1-Bromoethyl benzene

Living polystyrene macromonomer

(Non-end-functionalized macromonomer)

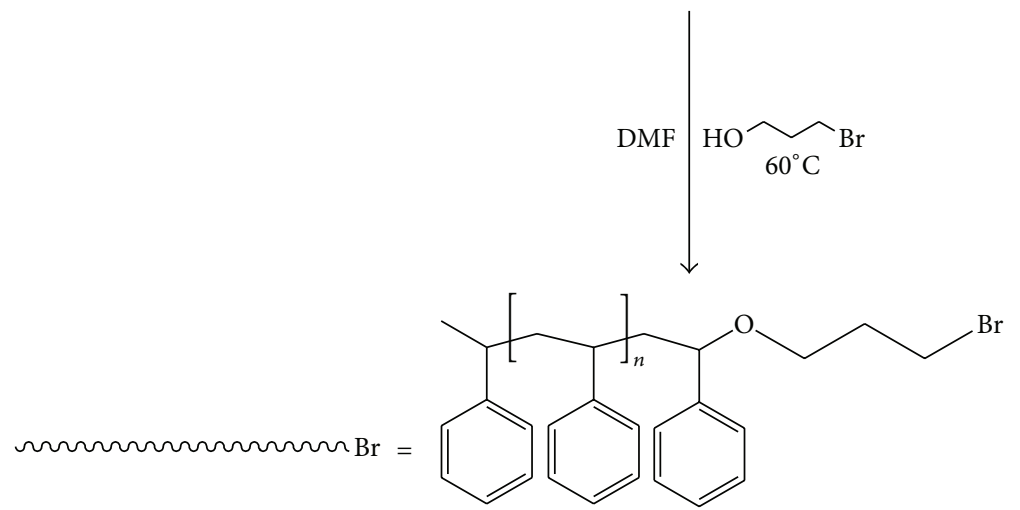

Primary alkyl brominated polystyrene

(End-functionalized macromonomer)

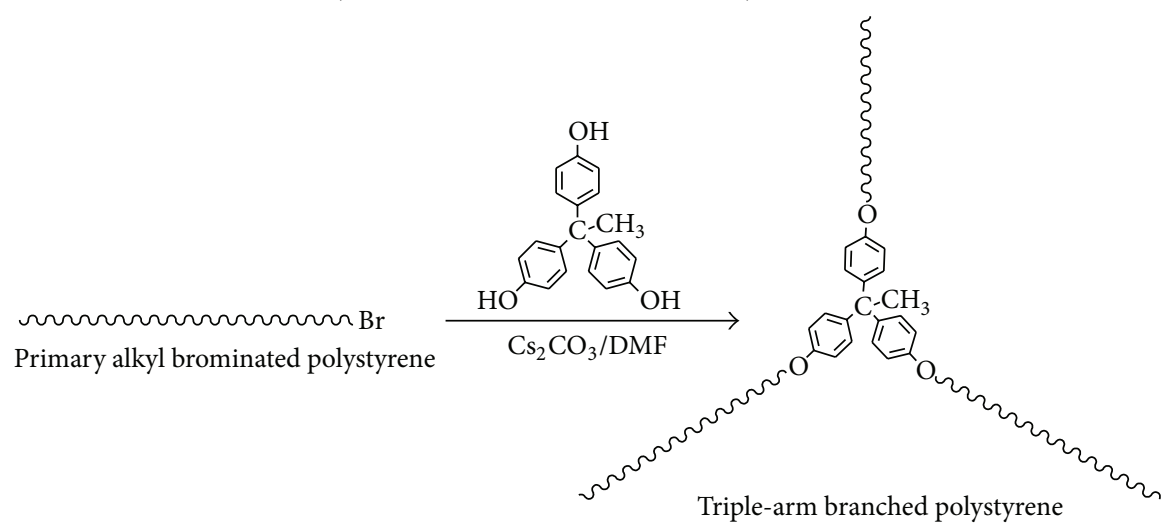

SCHEME 2: Synthesis of branched polystyrene after end functionalization.

fractionation using toluene and methanol as solvent and nonsolvent system, to remove uncoupled polymer which resulted in a distinct branched structure as apparent from the GPC analysis (Figure 4(d)). The resulting branched polymer has a low PDI (1.18) and is monomodal. The ether linkage in between the core molecule and linear polymeric chain was successfully formed as evident from the ${ }^{1} \mathrm{H}$ NMR spectrum (Figure 3). The complete shift of signal from $3.75 \mathrm{ppm}$ $\left(\mathrm{CH}_{2} \mathrm{Br}\right)$ to $3.95\left(\mathrm{CH}_{2} \mathrm{OPh}\right)$ has confirmed the transformation of bromine end group into ether linkage quantitatively. The molecular weight of the resultant branched polymer is approximately a triple more than the molecular weight of the unbranched alkylbrominated polystyrene which further confirms the efficient coupling.

The molecular weight obtained at different time intervals by coupling of non-end-functionalized polystyrene was plotted and compared with the molecular weight obtained by coupling of end-functionalized polystyrene. There was no significant increase in the molecular weight even after 48 hours when coupling was attempted without end functionalizing polystyrene as evident from graph (Figure 5). On the other hand, after successful insertion of the primary alkyl bromide moiety at the end of linear polymeric chain and its coupling with core molecule, the molecular weight starts to increase from the third hour of the reaction and coupling was assumed to be completed within 12 hours with no apparent increase in molecular weight thereafter. There is an excellent agreement between the molecular of the linear alkyl brominated polymer and the triarm star-branched polymer.

\section{Conclusions}

Well-defined triarm star-shaped polystyrene was successfully synthesised via ATRP using very simple and efficient methodology. ${ }^{1} \mathrm{H}$ NMR and GPC results have confirmed the formation of branched polymeric structure. The simplicity of 


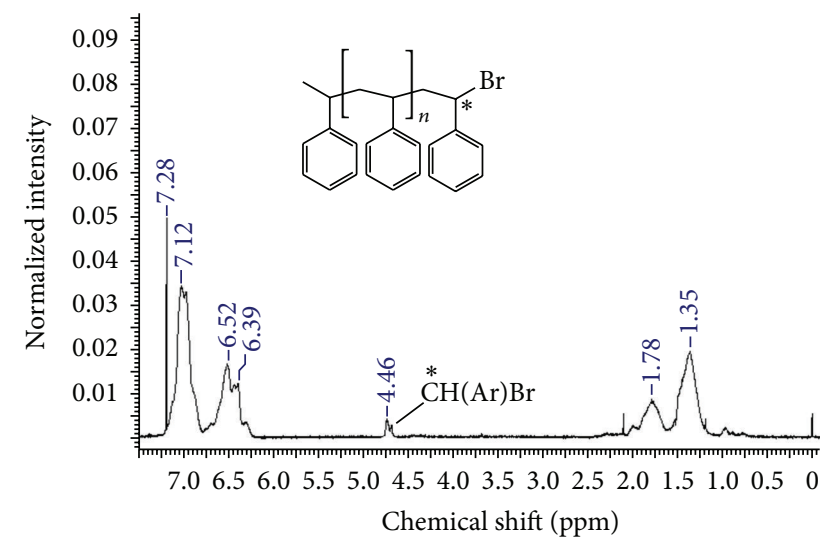

FIGURE 1: ${ }^{1} \mathrm{H}-\mathrm{NMR}$ spectra of non-end-functionalized polystyrene.

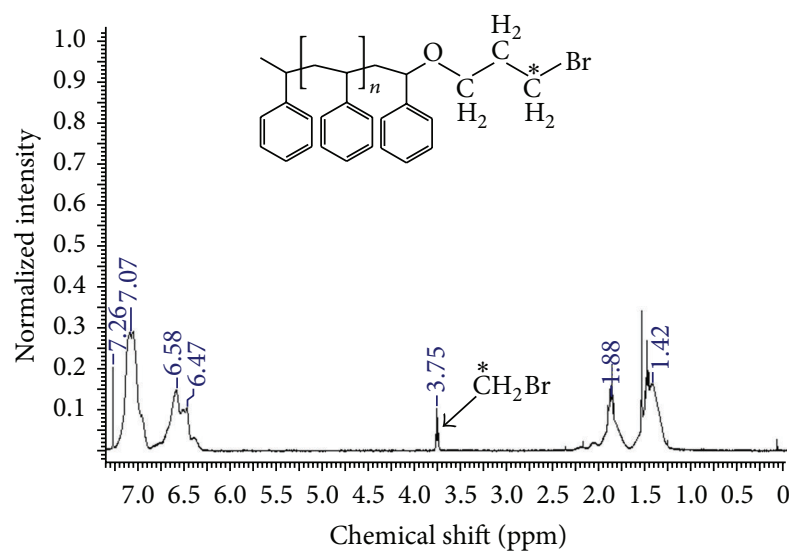

FIGURE 2: ${ }^{1} \mathrm{H}-\mathrm{NMR}$ spectra of end-functionalized polystyrene.

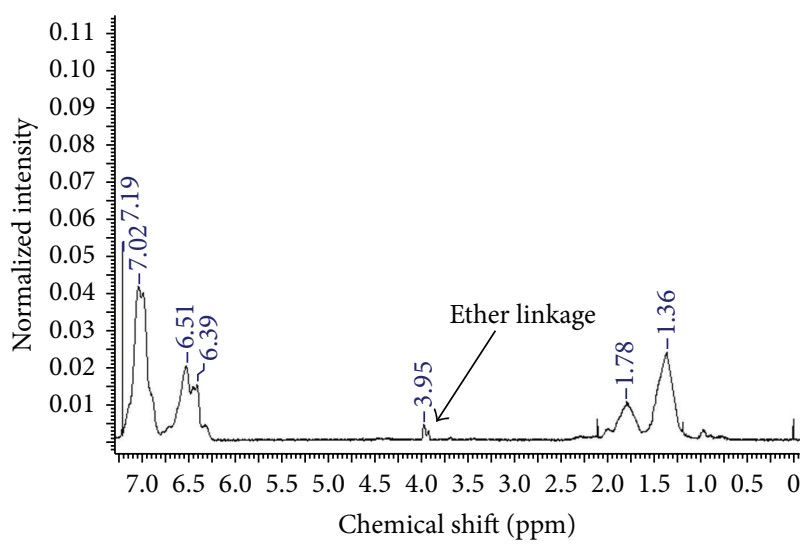

FIGURE $3:{ }^{1} \mathrm{H}-\mathrm{NMR}$ spectra of coupled polystyrene.

the reported methodology, commercial availability of the chemicals and good control over polymeric architecture, makes ATRP an important tool to fabricate controlled branched polymers. Highly branched polymers and copolymers with well-defined structure can be synthesised by modifying the initiators and monomers by exploiting this methodology. Further work is ongoing and is being explored

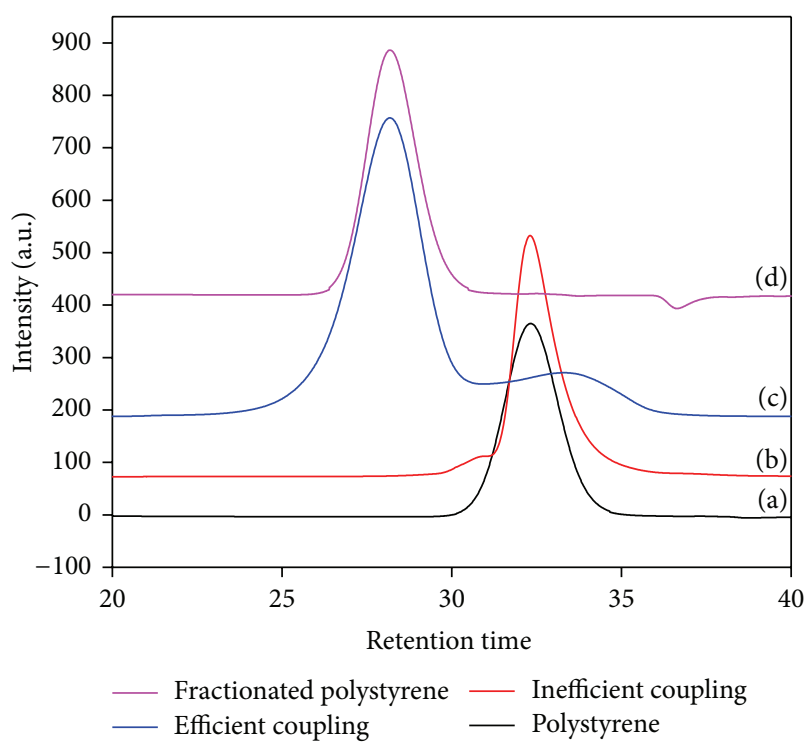

FIGURE 4: GPC traces of (a) polystyrene precursor macromonomer, $\left(\mathrm{Mn} 7455 \mathrm{~g} \cdot \mathrm{mol}^{-1}\right)$. (b) Coupled non-end-functionalized polystyrene. (c) Crude unfractionated coupled end functionalized polystyrene. (d) Coupled end functionalized polystyrene after fractionation $\left(\mathrm{Mn} 21436 \mathrm{~g} \cdot \mathrm{mol}^{-1}\right)$.

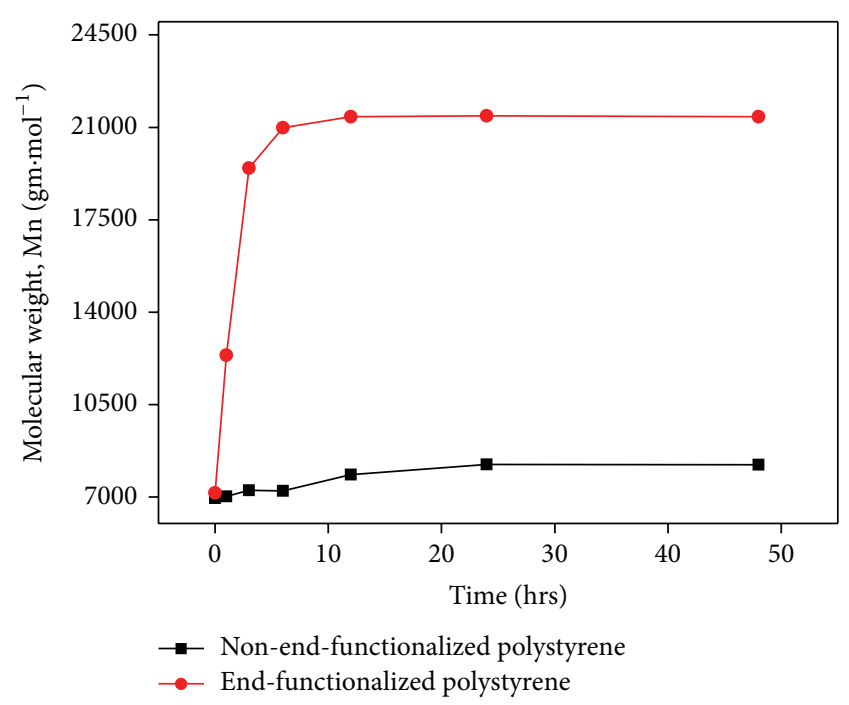

FIGURE 5: Molecular weight Mn versus time plot for coupling of non-end-functionalized and end-functionalized polystyrene macromonomer.

in our group where the results will be published in the near future.

\section{Conflict of Interests}

The authors declare that there is no conflict of interests regarding the publication of this paper. 


\section{Acknowledgment}

The authors would like to thank University of Malaya (UM) for the research facilities and financial support through HIR/MOHE/SC/F00031.

\section{References}

[1] K. Matyjaszewski and J. Xia, "Atom transfer radical polymerization," Chemical Reviews, vol. 101, no. 9, pp. 2921-2990, 2001.

[2] M. Kamigaito, T. Ando, and M. Sawamoto, "Metal-catalyzed living radical polymerization," Chemical Reviews, vol. 101, no. 12, pp. 3689-3745, 2001.

[3] D. Braun, "Origins and development of initiation of free radical polymerization processes," International Journal of Polymer Science, vol. 2009, Article ID 893234, 10 pages, 2009.

[4] M. K. Georges, R. P. N. Veregin, P. M. Kazmaier, and G. K. Hamer, "Narrow molecular weight resins by a free-radical polymerization process," Macromolecules, vol. 26, no. 11, pp. 2987-2988, 1993.

[5] N. V. Tsarevsky, T. Sarbu, B. Göbelt, and K. Matyjaszewski, "Synthesis of styrene-acrylonitrile copolymers and related block copolymers by atom transfer radical polymerization," Macromolecules, vol. 35, no. 16, pp. 6142-6148, 2002.

[6] M. Spasojević, J. Vorenkamp, M. Jansen, P. de Vos, and A. Schouten, "Synthesis and phase behavior of poly( $N$-isopropylacrylamide)-b-Poly(L-lysine hydrochloride) and poly(Nisopropylacrylamide-co-acrylamide)-b-poly(L-lysine hydrochloride)," Materials, vol. 7, no. 7, pp. 5305-5326, 2014.

[7] J.-S. Wang and K. Matyjaszewski, "Controlled/'living' radical polymerization. Atom transfer radical polymerization in the presence of transition-metal complexes," Journal of the American Chemical Society, vol. 117, no. 20, pp. 5614-5615, 1995.

[8] M. Rodlert, E. Harth, I. Rees, and C. J. Hawker, "End-group fidelity in nitroxide-mediated living free-radical polymerizations," Journal of Polymer Science Part A: Polymer Chemistry, vol. 38, pp. 4749-4763, 2000.

[9] M. Kato, M. Kamigaito, M. Sawamoto, and T. Higashimura, "Polymerization of methyl methacrylate with the carbon tetrachloride/dichlorotris-(triphenylphosphine) ruthenium (II)/ methylaluminum bis (2, 6-di-tert-butylphenoxide) initiating system: possibility of living radical polymerization," Macromolecules, vol. 28, no. 5, pp. 1721-1723, 1995.

[10] D. M. Haddleton, C. B. Jasieczek, M. J. Hannon, and A. J. Shooter, "Atom transfer radical polymerization of methyl methacrylate initiated by alkyl bromide and 2-pyridinecarbaldehyde imine copper(I) complexes," Macromolecules, vol. 30, no. 7, pp. 2190-2193, 1997.

[11] N. Hadjichristidis, M. Pitsikalis, S. Pispas, and H. Iatrou, "Polymers with complex architecture by living anionic polymerization," Chemical Reviews, vol. 101, no. 12, pp. 3747-3792, 2001.

[12] Y. Zhao, X. Shuai, C. Chen, and F. Xi, "Synthesis of star block copolymers from dendrimer initiators by combining ringopening polymerization and atom transfer radical polymerization," Macromolecules, vol. 37, no. 24, pp. 8854-8862, 2004.

[13] J. Bibiao, Y. Yang, D. Jian et al., "Preparation of hyperbranched polymers by atom transfer radical polymerization," Journal of Applied Polymer Science, vol. 83, no. 10, pp. 2114-2123, 2002.

[14] S. H. Yoo, J. H. Lee, J.-C. Lee, and J. Y. Jho, "Synthesis of hyperbranched polyacrylates in emulsion by atom transfer radical polymerization," Macromolecules, vol. 35, no. 4, pp. 11461148, 2002.
[15] A. Sunder, H. Frey, and R. Mülhaupt, "Hyperbranched polyglycerols by ring-opening multibranching polymerization," Macromolecular Symposia, vol. 153, no. 1, pp. 187-196, 2000.

[16] T. J. Smith and L. J. Mathias, "Hyperbranched poly(3-ethyl-3hydroxymethyloxetane) via anionic polymerization," Polymer, vol. 43, no. 26, pp. 7275-7278, 2002.

[17] A. Morita, H. Kudo, and T. Nishikubo, "Synthesis and chemical modification of hyperbranched polyethers with terminal hydroxy groups by the anionic ring-opening polymerization of 3-Alkyl-3-hydroxymethyl oxetanes," Journal of Polymer Science, Part A: Polymer Chemistry, vol. 42, no. 15, pp. 3739-3750, 2004.

[18] L. Wu, R. McHale, G. Feng, and X. Wang, "RAFT synthesis and self-assembly of free-base porphyrin cored star polymers," International Journal of Polymer Science, vol. 2011, Article ID 109693, 11 pages, 2011.

[19] S. Agostini and L. R. Hutchings, "Synthesis and temperature gradient interaction chromatography of model asymmetric star polymers by the 'macromonomer' approach," European Polymer Journal, vol. 49, no. 9, pp. 2769-2784, 2013.

[20] A. Hirao, A. Matsuo, and T. Watanabe, "Precise synthesis of dendrimer-like star-branched poly(methyl methacrylate)s up to seventh generation by an iterative divergent approach involving coupling and transformation reactions," Macromolecules, vol. 38, no. 21, pp. 8701-8711, 2005.

[21] P. F. W. Simon, W. Radke, and A. H. E. Müller, "Hyperbranched methacrylates by self-condensing group transfer polymerization," Macromolecular Rapid Communications, vol. 18, no. 9, pp. 865-873, 1997.

[22] L. R. Hutchings and S. J. Roberts-Bleming, "DendriMacs. welldefined dendritically branched polymers synthesized by an iterative convergent strategy involving the coupling reaction of AB2 macromonomers," Macromolecules, vol. 39, no. 6, pp. 21442152, 2006.

[23] C. N. Urban, C. A. Bell, M. R. Whittaker, and M. J. Monteiro, "Convergent synthesis of second generation AB-type miktoarm dendrimers using "click" chemistry catalyzed by copper wire," Macromolecules, vol. 41, no. 4, pp. 1057-1060, 2008.

[24] G. Wang, X. Luo, Y. Zhang, and J. Huang, "Synthesis of dendrimer-like copolymers based on the star[PolystyrenePoly(ethylene oxide)-Poly(ethoxyethyl glycidyl ether)] terpolymers by click chemistry," Journal of Polymer Science Part A: Polymer Chemistry, vol. 47, no. 18, pp. 4800-4810, 2009.

[25] N. Clarke, E. D. Luca, J. M. Dodds, S. M. Kimani, and L. R. Hutchings, "HyperMacs-long chain hyperbranched polymers: a dramatically improved synthesis and qualitative rheological analysis," European Polymer Journal, vol. 44, no. 3, pp. 665-676, 2008.

[26] L. R. Hutchings, "DendriMacs and HyperMacs-emerging as more than just model branched polymers," Soft Matter, vol. 4, no. 11, pp. 2150-2159, 2008.

[27] A. Matsuo, T. Watanabe, and A. Hirao, "Synthesis of welldefined dendrimer-like branched polymers and block copolymer by the iterative approach involving coupling reaction of living anionic polymer and functionalization," Macromolecules, vol. 37, no. 17, pp. 6283-6290, 2004.

[28] S. Angot, D. Taton, and Y. Gnanou, "Amphiphilic stars and dendrimer-like architectures based on poly(ethylene oxide) and polystyrene," Macromolecules, vol. 33, no. 15, pp. 5418-5426, 2000.

[29] B. Lepoittevin, R. Matmour, R. Francis, D. Taton, and Y. Gnanou, "Synthesis of dendrimer-like polystyrene by atom 
transfer radical polymerization and investigation of their viscosity behavior," Macromolecules, vol. 38, no. 8, pp. 3120-3128, 2005.

[30] I. Chalari and N. Hadjichristidis, "Synthesis of well-defined second-generation dendritic polymers of isoprene (I) and styrene $(\mathrm{S}):\left(\mathrm{S}_{2} \mathrm{I}\right)_{3},\left(\mathrm{SI}^{\prime} \mathrm{I}\right)_{3},\left(\mathrm{I}^{\prime \prime} \mathrm{I}^{\prime} \mathrm{I}\right)_{3}$, and $\left(\mathrm{I}_{2}^{\prime} \mathrm{I}\right)_{4}$," Journal of Polymer Science, Part A: Polymer Chemistry, vol. 40, no. 10, pp. 1519-1526, 2002.

[31] K. Orfanou, H. Iatrou, D. J. Lohse, and N. Hadjichristidis, "Synthesis of well-defined second (G-2) and third (G-3) generation dendritic polybutadienes," Macromolecules, vol. 39, no. 13, pp. 4361-4365, 2006.

[32] M. Trollsås and J. L. Hedrick, "Dendrimer-like star polymers," Journal of the American Chemical Society, vol. 120, no. 19, pp. 4644-4651, 1998.

[33] S. Pascual, B. Coutin, M. Tardi, A. F. Polton, and J.-P. Vairon, "Homogeneous atom transfer radical polymerization of styrene initiated by 1-chloro-1-phenylethane/copper (I) chloride/bipyridine in the presence of dimethylformamide," Macromolecules, vol. 32, no. 5, pp. 1432-1437, 1999.

[34] V. Coessens and K. Matyjaszewski, "End group transformation of polymers prepared by ATRP, substitution to azides," Journal of Macromolecular Science-Pure and Applied Chemistry, vol. 36, no. 5-6, pp. 667-679, 1999.

[35] L. G. Wade, "Reaction of alcohols," in Organic Chemistry, R. M. John Challice, Ed., pp. 494-495, Pearson Education, Upper Saddle River, NJ, USA, 6th edition, 2006. 

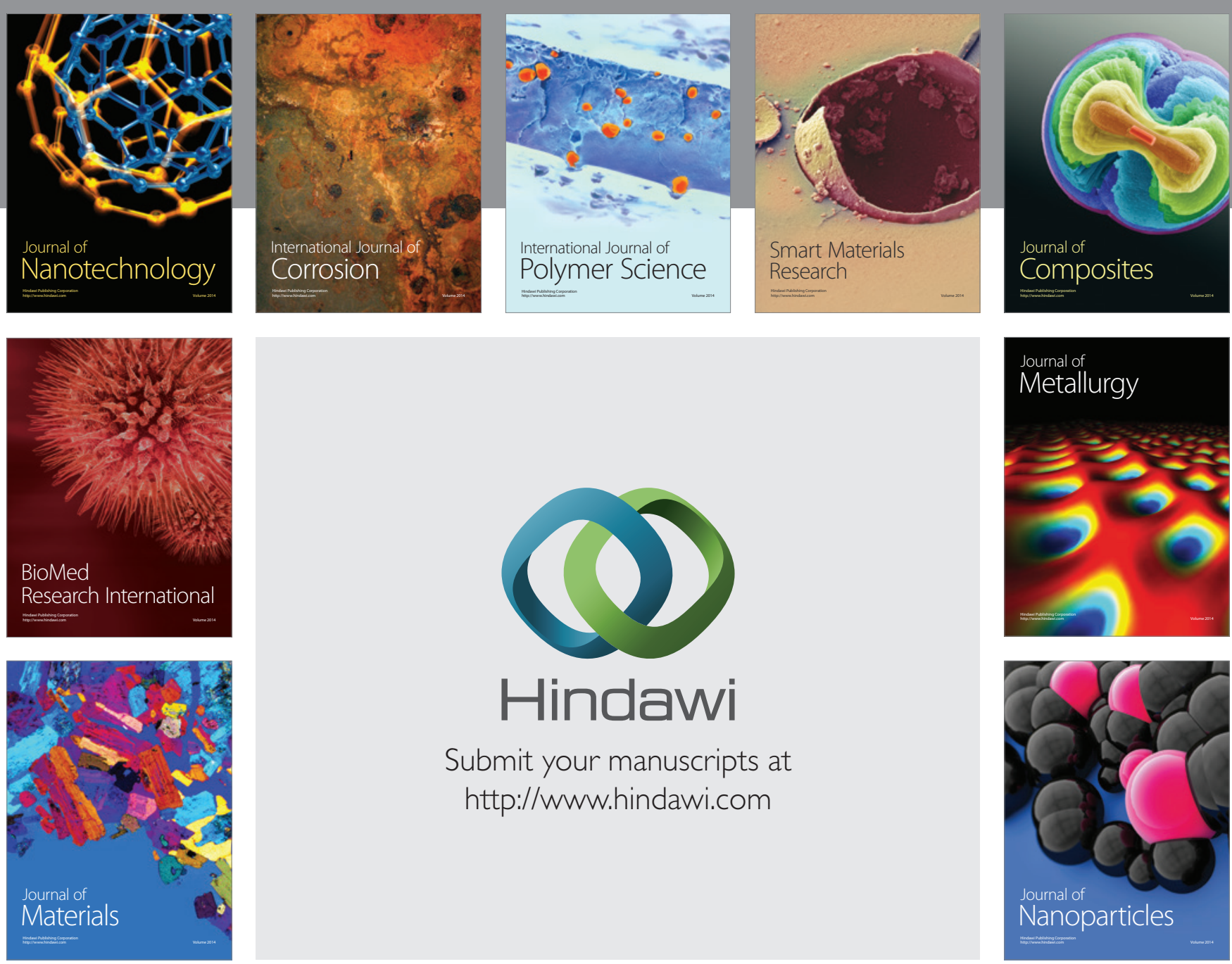

Submit your manuscripts at http://www.hindawi.com
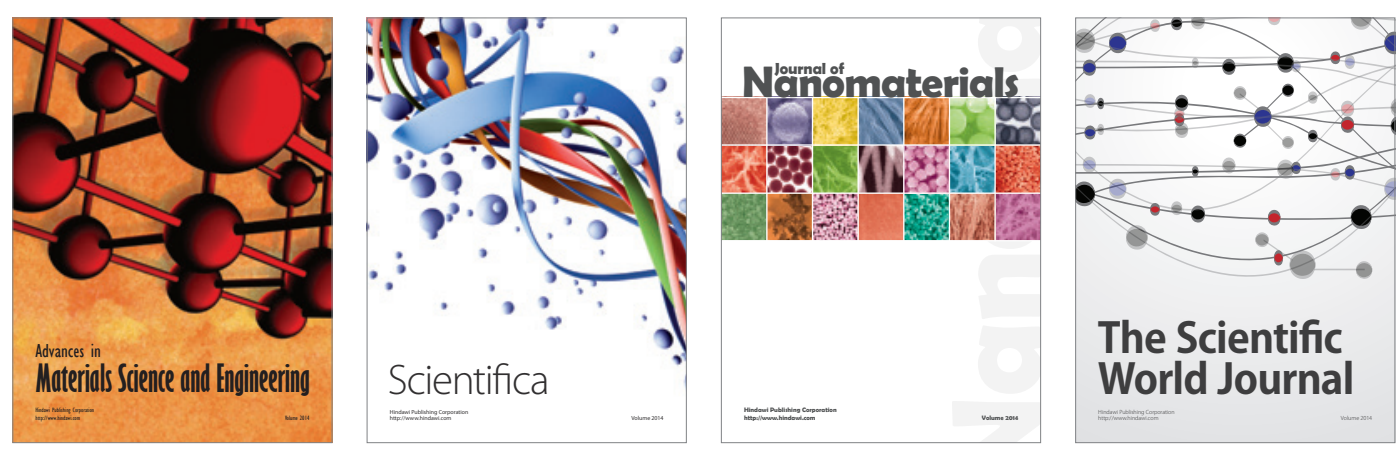

\section{The Scientific World Journal}
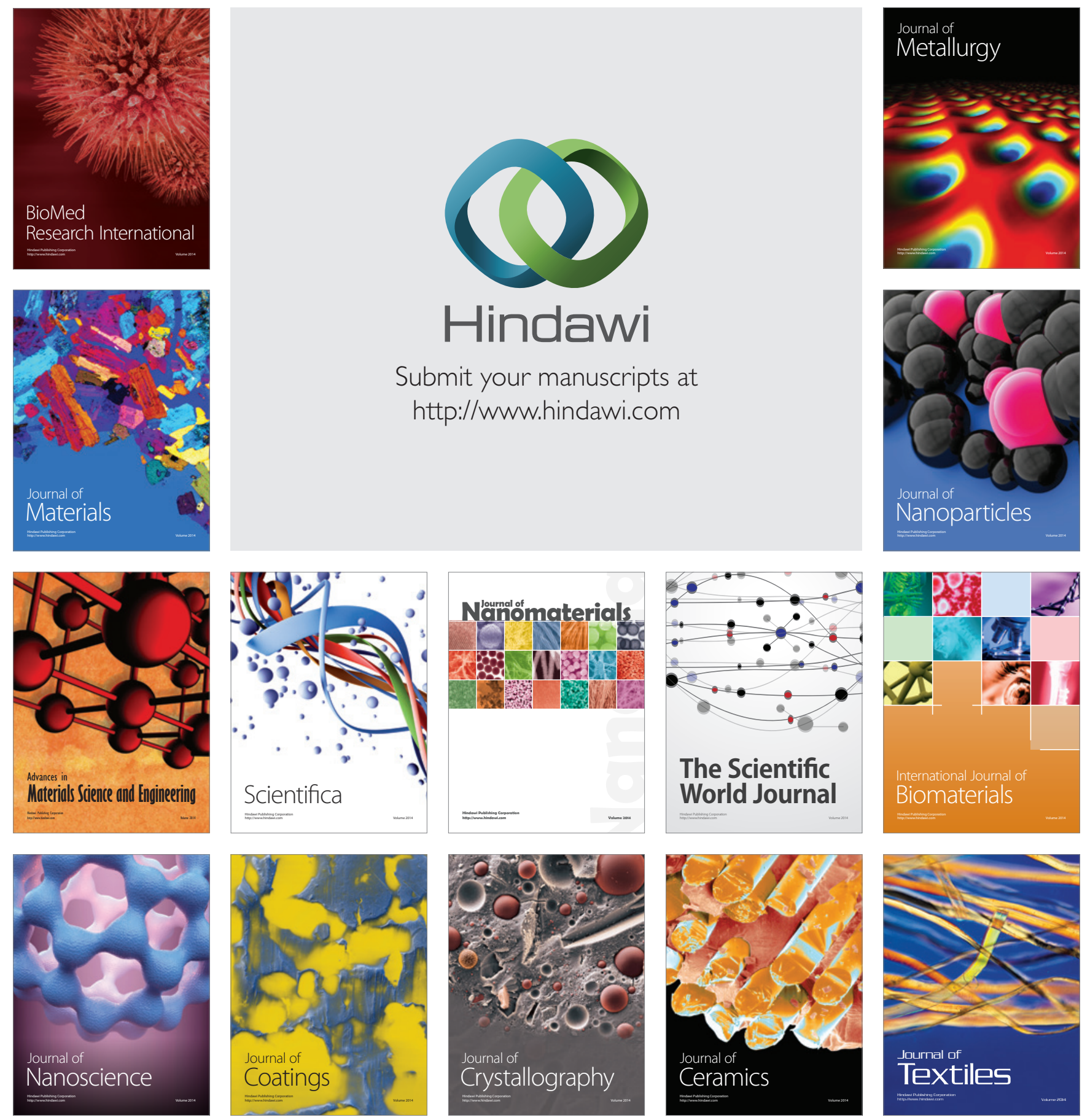